Research Paper

\title{
MicroRNA Profile of Human Bone Marrow Mesenchymal Stem Cells during Hepatic Differentiation and Therapy
}

\author{
Jing Jiang ${ }^{1}$, Jiaojiao Xin ${ }^{1}$, Wenchao Ding ${ }^{1}$, Dongyan Shi ${ }^{1}$, Suwan Sun ${ }^{1}$, Beibei Guo ${ }^{1}$, Xingping Zhou ${ }^{1}$, Chufan \\ Zheng', Jun $\mathrm{Li}^{1}{ }^{\square}$ \\ 1. State Key Laboratory for Diagnosis and Treatment of Infectious Diseases, Collaborative Innovation Center for Diagnosis and Treatment of Infectious \\ Diseases, The First Affiliated Hospital, Zhejiang University School of Medicine, 79 Qingchun Rd., Hangzhou, 310003, China. \\ 2. Hangzhou No.14 High School, 580 Fengqi Rd, Gongshu District, Hangzhou, 310006, China. \\ $\bowtie$ Corresponding author: Jun Li, Ph.D. \& M.D., Professor, Tel.: 86-571-87236425; Fax: 86-571-87236425; E-mail: lijun2009@zju.edu.cn.
}

(c) The author(s). This is an open access article distributed under the terms of the Creative Commons Attribution License (https://creativecommons.org/licenses/by/4.0/). See http://ivyspring.com/terms for full terms and conditions.

Received: 2021.10.01; Accepted: 2021.11.26; Published: 2022.01.01

\begin{abstract}
Background and Aims: MicroRNAs (miRNAs) play important roles in hepatocyte differentiation from human bone marrow mesenchymal stem cells (hBMSCs) and the therapeutic application in vivo. However, the mechanisms of miRNA regulation are still unknown. This study aimed to profile the miRNA basis for improving the function of hBMSC-differentiated hepatocyte-like cells (hBMSC-Heps).

Methods: Characteristic miRNAs of hBMSC-Heps were identified by transcriptome sequencing and validated by quantitative real-time polymerase chain reaction (qRT-PCR). An in vivo hBMSC transplantation model was used to assess the regulatory effects of miRNAs on liver regeneration during hBMSC therapy in pigs with fulminant hepatic failure (FHF). The biological functions of significant miRNA molecules were confirmed by transfection of miRNA activators or inhibitors into hBMSCs during hepatogenic differentiation.

Results: The transcriptome of hBMSC-Heps showed characteristics distinct from those of undifferentiated hBMSCs. A total of 77 miRNAs were significantly differentially expressed in hBMSC-Heps at day 10 and day 20 after hBMSC differentiation that were directly related to the functions of hepatocytes. Among the top 10 significantly differentially expressed and the top 10 most abundant miRNAs, nine miRNAs that exhibited a pattern of gradual change were chosen for further analysis. The expression of nine miRNAs was confirmed by qRT-PCR in vitro and showed the same changing trends in vivo in an hBMSC transplantation model in pigs. Functional experiments with these miRNAs showed that activators of hsa-miR-26b-5p and hsa-miR-148a-3p and an inhibitor of hsa-miR-423-3p were sufficient to improve the differentiation of hBMSCs into hepatocyte-like cells.

Conclusions: Transcriptome profiles of miRNA revealed the basis of the differentiation and development of hBMSC-Heps. Manipulation of three miRNAs (hsa-miR-26b-5p, hsa-miR-148a-3p and hsa-miR-423-3p) significantly improved hepatocyte generation and liver regeneration, indicating the potential of these miRNAs for future clinical applications.
\end{abstract}

Key words: microRNA, human bone marrow mesenchymal stem cells; hepatic differentiation; fulminant hepatic failure

\section{Introduction}

Fulminant hepatic failure (FHF) is a lethal syndrome that involves the acute deterioration of hepatic and extrahepatic organs which associated with high mortality $[1,2]$. Liver transplantation is the only option for saving patients with FHF, but transplantation is limited by the shortage of donors and by contraindications [3]. Among several strategies developed to overcome this situation, stem cell-based therapies hold substantial promise $[4,5,6]$. The hepatic differentiation ability of stem cells has been characterized in vivo and in vitro in many previous studies from our laboratory and others, and the results suggest that this strategy is an excellent candidate for the treatment of FHF and end-stage liver disease $[7,8,9,10]$. Our previous study demonstrated that transplantation of human bone marrow mesenchymal stem cells (hBMSCs) can rescue FHF pigs through stem cells proliferation and differentiation into hepatocytes [9]. As an alternative, hBMSC-derived hepatocyte-like cells (hBMSC-Heps) 
serve as a potential source for hepatocyte transplantation and bioartificial liver systems [7, 11]. Although the ability of hBMSCs to differentiate into hepatocytes in vitro and in vivo has been widely recognized, most studies have focused on the therapeutic promise of stem cells; the mechanism of hBMSC differentiation and the performance of hBMSC-Heps are still unclear. A systematic evaluation of hBMSC-Heps is needed to pave the way for their future clinical application by revealing their distinct functions and assessing potential risks.

Recently, microRNAs (miRNAs) have attracted great attention because they regulate up to $30 \%$ of human genes, and their influence underlies almost every aspect of biological processes at both the transcriptional and posttranscriptional levels. miRNAs are the most abundant class of small endogenous noncoding regulatory RNA molecules and generally function by inhibiting gene expression or silencing genes through destabilization and degradation of their target mRNA [12, 13]. Most miRNAs are evolutionarily conserved but exhibit developmental timing and tissue specificity [14, 15]. Many studies have demonstrated that miRNAs play an important role in several physiological and pathological processes in the liver [16] and have profound effects on biological processes such as cell development, differentiation and proliferation. The expression of specific miRNAs at different stages during early human liver development [17, 18] and during hepatic regeneration following partial hepatectomy [19] has been well described. As key regulators controlling cell fate and tissue development, miRNAs might serve as efficient molecular switches that control the fate of stem cells $[16,20]$. Studies have also addressed the function of miRNAs in liver development, showing that the expression of miRNAs plays a decisive role in stem cells differentiation into hepatocytes or cholangiocytes [21] and promotes hepatic differentiation $[22,23]$. Recently, large-scale miRNA expression profiling of stem cells has become a major focus of research efforts. Studies have revealed some specific expression signatures of hepatic differentiation through miRNA arrays of human embryonic stem cells [24] and umbilical cord mesenchymal-derived stem cells [25], but hBMSCs have not been investigated in this way. However, these studies have been limited to comparing the undifferentiated and differentiated states of stem cells in vitro. The differences between hBMSC-Heps and primary human hepatocytes (PHHs) and the details of the differentiation process in vitro and in vivo are still unclear.

In this study, the miRNA basis of hBMSC- derived hepatocyte-like cells was compared with that of PHHs and validated in vivo with an hBMSC transplantation model in FHF pigs. Overall, we found three miRNA molecules that can improve hepatic differentiation and generate more functional hBMSCHeps for future treatment of various liver diseases.

\section{Methods}

\section{Cell isolation, culture and phenotypic identification}

HBMSCs were isolated from bone marrow that was collected from the iliac crest of 4 healthy volunteers whom given informed written consent. The hBMSCs derived from each volunteer were isolated as previously described [26]. Phenotypic identification of cultured hBMSCs was performed by standard flow cytometry. hBMSCs (passage $3-7,1 \times 10^{6}$ cells) were incubated for $60 \mathrm{~min}$ with PE- or FITC-conjugated mouse monoclonal antibodies, namely, anti-human CD29, anti-human CD45 (Abcam, Cambridge, UK), anti-human CD34 (Santa Cruz Biotechnology, Santa Cruz, CA, USA), or anti-human CD90 (BD Biosciences, San Jose, CA, USA), or with an immunoglobulin isotype as a negative control. The cells were analyzed with a FACS Calibur system (FC500, Beckman Coulter, Fullerton, CA, USA) after washing. PHHs were isolated using a four-step collagenase perfusion method as previously described [27]. Briefly, the liver segments were perfused by the extracorporeal circulating perfusion apparatus with four different buffer solutions supplemented with $0.37 \mathrm{mg} / \mathrm{ml}$ EDTA, $0.5 \%$ dispase, $0.05 \%$ collagenase type IV (Gibco BRL, Grand Island, $\mathrm{NY}$ ), and $40 \mu \mathrm{g} / \mathrm{ml}$ DNase I (Sigma, St. Louis, MO). The remnants were dispersed mechanically with a scalpel blade and filtered sequentially through a 100 $\mu \mathrm{m}$ nylon mesh. The isolated cells were harvested and centrifuged at $4{ }^{\circ} \mathrm{C}$ and $50 \mathrm{~g}$ for $3 \mathrm{~min}$.

\section{Hepatic differentiation of hBMSCs}

Hepatic differentiation of harvested hBMSCs was performed according to the methods described in our previous study [8, 9]. Briefly, hBMSCs (third passage, $1 \times 10^{6}$ cells) were cultured with hepatocyte differentiation basal medium containing $20 \mathrm{ng} / \mathrm{ml}$ human hepatocyte growth factor (hHGF); $20 \mu \mathrm{g} / \mathrm{ml}$ dexamethasone; $50 \mathrm{mg} / \mathrm{ml}$ insulin, transferrin, and selenium (ITS) premix; and $100 \mathrm{U} / \mathrm{ml}$ penicillin and $100 \mu \mathrm{g} / \mathrm{ml}$ streptomycin for 2 weeks. After that, the cells were cultured in another medium including 20 $\mathrm{ng} / \mathrm{ml}$ oncostatin M (OSM), $20 \mu \mathrm{g} / \mathrm{ml}$ dexamethasone, $50 \mathrm{mg} / \mathrm{ml}$ ITS premix, $100 \mathrm{U} / \mathrm{ml}$ penicillin and $100 \mu \mathrm{g} / \mathrm{ml}$ streptomycin for an additional week. Hepatic functions were 
characterized prior to hBMSC differentiation and then at 10 and 20 days after differentiation. The expression of liver-specific genes (amplified by the primer sequences listed in Table S1) was analyzed by qRT-PCR, albumin (ALB) expression was measured by immunostaining, and glycogen synthesis was analyzed by periodic acid-Schiff (PAS) staining system (Sigma, St. Louis, MO) using methods described previously [8]. Briefly, the fixed specimen slides immersed in Schiff's reagent for $15 \mathrm{~min}$ at room temperature and rinsed in running tap water for 5 min. After that, the slides were counterstained in Hematoxylin solution for $90 \mathrm{~s}$, washed in running tap water for 15-30 s, dried and examined microscopically.

\section{hBMSC transplantation in an FHF pig model}

An in-house translational model was used as previously described [9]. Briefly, an FHF pig model was induced with D-galactosamine $(1.5 \mathrm{~g} / \mathrm{kg}$ body weight) via jugular vein catheterization of male Chinese miniature experimental pigs (Taihe Biotechnology, Jiangsu, China) weighing $8-10 \mathrm{~kg}$ and aged 2.5 months. The FHF pigs were randomized into two groups: an hBMSC intraportal transplantation group that received a transfusion of hBMSCs $\left(3 \times 10^{6} / \mathrm{kg}\right.$, suspended in $10 \mathrm{ml}$ of normal saline) via the intrahepatic portal vein under B-ultrasound guidance and a control group that underwent a sham procedure with an equal volume of normal saline without cells. All transplantation operations were performed by the same B-ultrasound expert with more than 5 years of experience.

\section{RNA isolation and miRNA sequencing (miRNA-seq)}

Total RNA was extracted and purified from cells with TRIzol reagent (Invitrogen, CA, USA) following the manufacturer's instructions. The cell samples included undifferentiated hBMSCs (day 0; D0 group) and hBMSC-Heps at 10 days (D10 group) and 20 days (D20 group) after hepatic differentiation ( $n=4$ /group), with freshly isolated PHHs as positive controls $(n=3)$. A sequencing library was prepared using the Illumina TruSeq ${ }^{\mathrm{TM}}$ Small RNA Sample Preparation Guide; the steps included adapter ligation, reverse transcription, PCR amplification, and gel purification. Deep sequencing of small RNAs was performed using an Illumina HiSeq 2000 sequencing system (Illumina, San Diego, CA, USA). The average number of sequencing reads was approximately 10 million per RNA sample.

\section{Bioinformatics analysis of sequencing data}

Prior to further analysis, the sequencing reads were preprocessed by cutting the $3^{\prime}$ adapter sequence using the cut-adapt program. After clipping, the sequence reads shorter than 18 nucleotides were removed. Furthermore, a dataset that consisted of unique sequences with their associated read counts was obtained by removing the identical sequences and recording the remaining reads. To improve the efficiency and reliability of the mapping steps, the singleton reads were not included in subsequent analyses. The obtained sequences were aligned to the human genome (hg19) downloaded from the University of California, Santa Cruz (UCSC) genome database with the program miRDeep2 and mapped against miRNA precursor sequences from miRBase release v21 [28]. For each sample, the read counts of the miRNAs were calculated and recorded.

\section{Validation of miRNAs in vitro and in vivo}

Liver tissues were collected from the experimental pigs in the normal group (PN), FHF pigs in control group at day 3 after D-galactosamine induction (PC-D3), and pigs in hBMSCs transplantation group at week 3 and week 5 (PT-3W/PT-5W). To determine the effect of the transplanted hBMSCs on liver regeneration, liver tissues were analyzed using Hematoxylin and eosin (H\&E) staining and immunohistochemistry with the human hepatocyte-specific marker ALB (Bethyl, Texas, USA) and an HSA (Abcam, Cambridge, UK). For H\&E staining, the liver tissue section was heat-fixed at $60^{\circ} \mathrm{C}$ for 1 hour and stained with $\mathrm{H} \& \mathrm{E}$ as described previously [9].

Selected genes, including liver-specific genes and significantly differentially expressed miRNAs from the miRNA-seq experiments, were further validated by qRT-PCR using an ABI $7500 \mathrm{HT}$ instrument (Applied Biosystems, Waltham, MA, USA), as described previously [29]. The qRT-PCR was performed with a two-step protocol following the manufacturer's instructions. Briefly, total RNA was reverse transcribed with a miRNA-specific primer, and then qRT-PCR was performed with TaqMan probes (Invitrogen, CA, USA). The target genes were assayed in triplicate on each plate. Significantly differentially expressed miRNAs in the above cell and tissue samples were selected for qRT-PCR validation.

\section{Target prediction of differentially expressed miRNAs and GO enrichment analysis}

The target genes of the selected miRNAs were predicted using the database miRDB, which collects miRNA-target interactions predicted based on support vector machines and high-throughput training datasets. All targets were downloaded from the website with Homo sapiens as the predicted species. To identify the regulatory functions of the miRNAs, Gene ontology (GO) annotation was 
performed for the target genes using ClueGO [30] in Cytoscape, which provided functional information on the gene products described with domain-specific ontologies.

\section{Transfection of hBMSCs during hepatic differentiation}

Activators and inhibitors of nine selected miRNAs (hsa-miR-203a-3p, hsa-miR-204-5p, hsa-miR101-3p, hsa-miR-26b-5p, hsa-miR-148a-3p, hsa-miR93-3p, hsa-miR-423-3p, hsa-miR-222-3p, and hsa-miR224-5p) were synthesized by GenePharma Co., Ltd. (Table S2). hBMSCs (third passage) were cultured in T25 flasks. When the cultured cells reached 70\% confluence, a Targefect transfection kit [31] (Targeting Systems) was used to transfect the cells with $25 \mathrm{nM}$ scrambled RNA, miRNA mimics (activators), or miRNA inhibitors. The transfection medium was changed to hepatic differentiation medium at 14 hours after transfection.

\section{Statistical analyses}

The differential expression of miRNAs among undifferentiated hBMSCs (day 0), hBMSC-Heps at 10 and 20 days after hepatic differentiation, and $\mathrm{PHHs}$ was analyzed with the DESeq2 package in the $R$ programming language. Significantly differentially expressed miRNAs were identified based on their logarithmic fold changes and $\mathrm{P}$ values. Unsupervised hierarchical clustering of the significantly differentially expressed miRNAs from the different groups was performed using the 'heatmap.2' function in the gplots package. This study employed the one-way analysis of variance (ANOVA) to analyze the significance in experiments contained more than two groups with the Tukey's test used for multiple comparisons. The p-value less than 0.05 was considered to indicate a significant difference. The results of the measurements are presented as the mean $\pm S D$, unless otherwise noted.

\section{Results}

\section{Phenotype and characteristics of hBMSC-Heps}

To identify the phenotype of the hBMSCs, third-passage hBMSCs were characterized by flow cytometry (Fig. 1A). The results showed that the hBMSCs used in this study were positive for CD29 $(97.8 \pm 1.7 \%)$ and CD90 $(96.3 \pm 1.7 \%)$ but negative for CD34 $(2.5 \pm 1.6 \%)$ and CD45 $(1.4 \pm 1.0 \%)$, suggesting that the cells used for further research possessed properties of typical hBMSCs. The phenotype of hBMSCs-Heps was determined by quantitative real-time polymerase chain reaction (qRT-PCR), immunocytochemistry and PAS staining. The qRT-PCR results for hepatocyte-specific genes
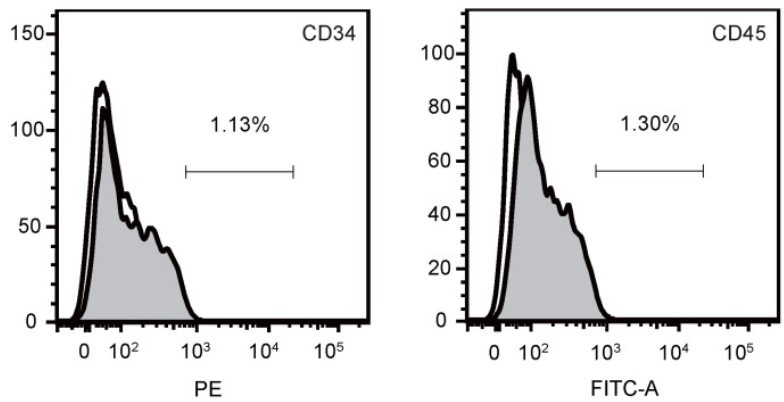

DO

C
B

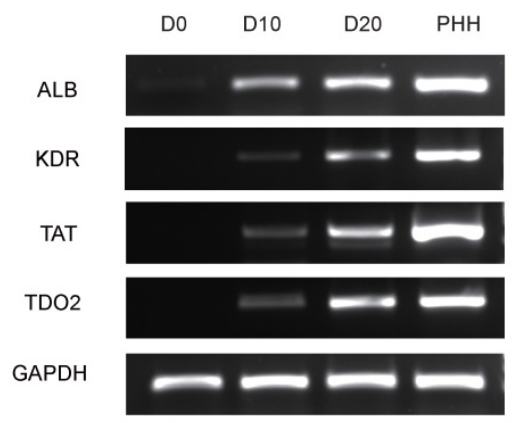

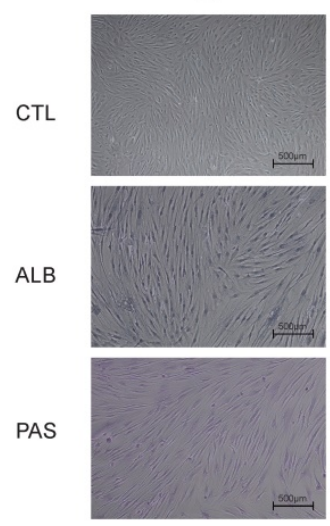

D10

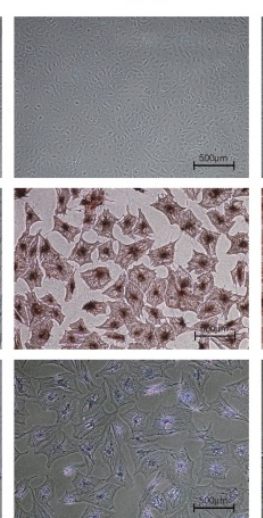

D20

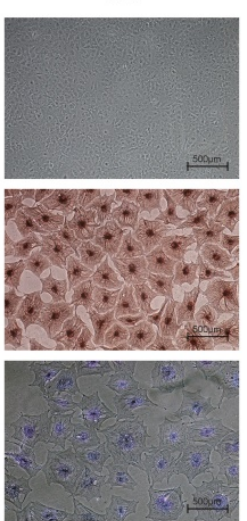

Figure 1. (A) Representative figures of flow cytometry analysis of CD29, CD90, CD34 and CD45. Third-passage hBMSCs were positive for CD29 (97.8 $\pm 1.7 \%$ ) and CD90 $(96.3 \pm 1.7 \%)$ but negative for CD34 $(2.5 \pm 1.6 \%)$ and CD45 $(1.4 \pm 1.0 \%), n=3 /$ group. (B) PCR analysis of the expression of ALB, KDR, TAT and TDO2 in hBMSCs (D0) and hepatic-differentiated hBMSCs (D10, D20). PHHs were used as positive controls. (C) Phase-contrast morphology and immunocytochemistry with ALB and PAS staining in undifferentiated hBMSCs (D0) and hBMSC-Heps (D10, D20); scale bar: $500 \mu \mathrm{m}$. 
showed that the expression levels of ALB, kinase insert domain receptor (KDR), tyrosine aminotransferase (TAT) and tryptophan 2,3-dioxygenase (TDO2) increased at D10 and reached higher levels at D20 after differentiation; undifferentiated hBMSCs were used as negative controls, and PHHs were used as positive controls (Fig. 1B). Under phase-contrast microscopy, hBMSC-Heps showed hepatocyte-like polygonal morphology with a low cytoplasm-tonucleus ratio, whereas hBMSCs exhibited a fibroblastlike morphology. Immunocytochemistry and PAS staining showed that the hBMSCs-Heps were positive for ALB and glycogen at D10 and D20 (Fig. 1C); undifferentiated hBMSCs (D0) were used as negative controls. The above results indicated that the stem cells had typical hBMSC phenotypes, and after hepatic differentiation following an accepted protocol, the hBMSC-Heps showed hepatocyte-like cellular characteristics in terms of morphology and the expression of typical mature hepatocyte markers. To

A

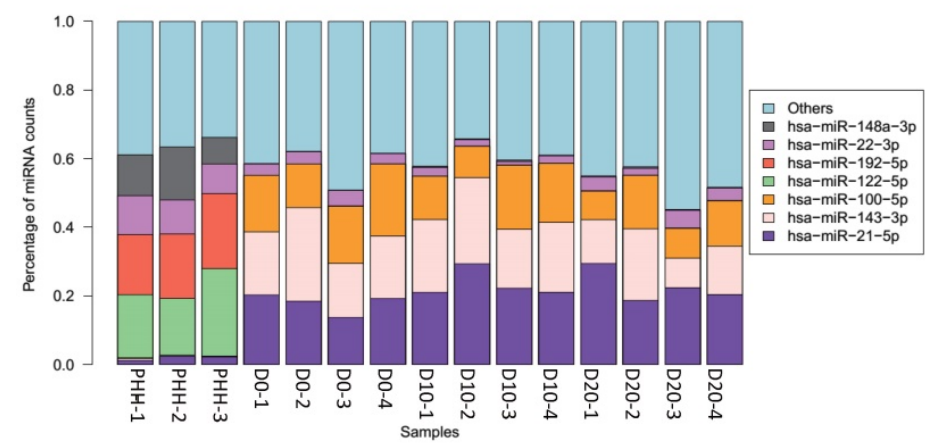

obtain the miRNA expression profiles of hBMSCs during different hepatic differentiation stages and in PHHs, miRNA sequencing was performed on undifferentiated hBMSCs (D0), hBMSC-Heps at D10 and D20, and PHHs. Based on the results of highsensitivity sequencing, 1150 miRNAs were detected in at least one sample, and 837, 845, 861 and 813 miRNAs in miRBase v21 were identified in at least one sample of the D0 hBMSCs, D10 hBMSC-Heps, D20 hBMSCHeps, and PHHs, respectively (Table S3).

\section{Qualitative analysis of differentially expressed miRNAs in hBMSC-Heps and PHHs}

In all hBMSCs and their hepatic differentiation samples, hsa-miR-21-5p was the most abundant miRNA, with a high percentage that ranged from $13.7 \%$ to $29.4 \%$ of the total known miRNAs in each sample. However, hsa-miR-21-5p constituted only $1 \%$ or $2 \%$ of the miRNAs in the PHHs (Fig. 2A). The differential expression of miRNAs in hBMSC-Heps

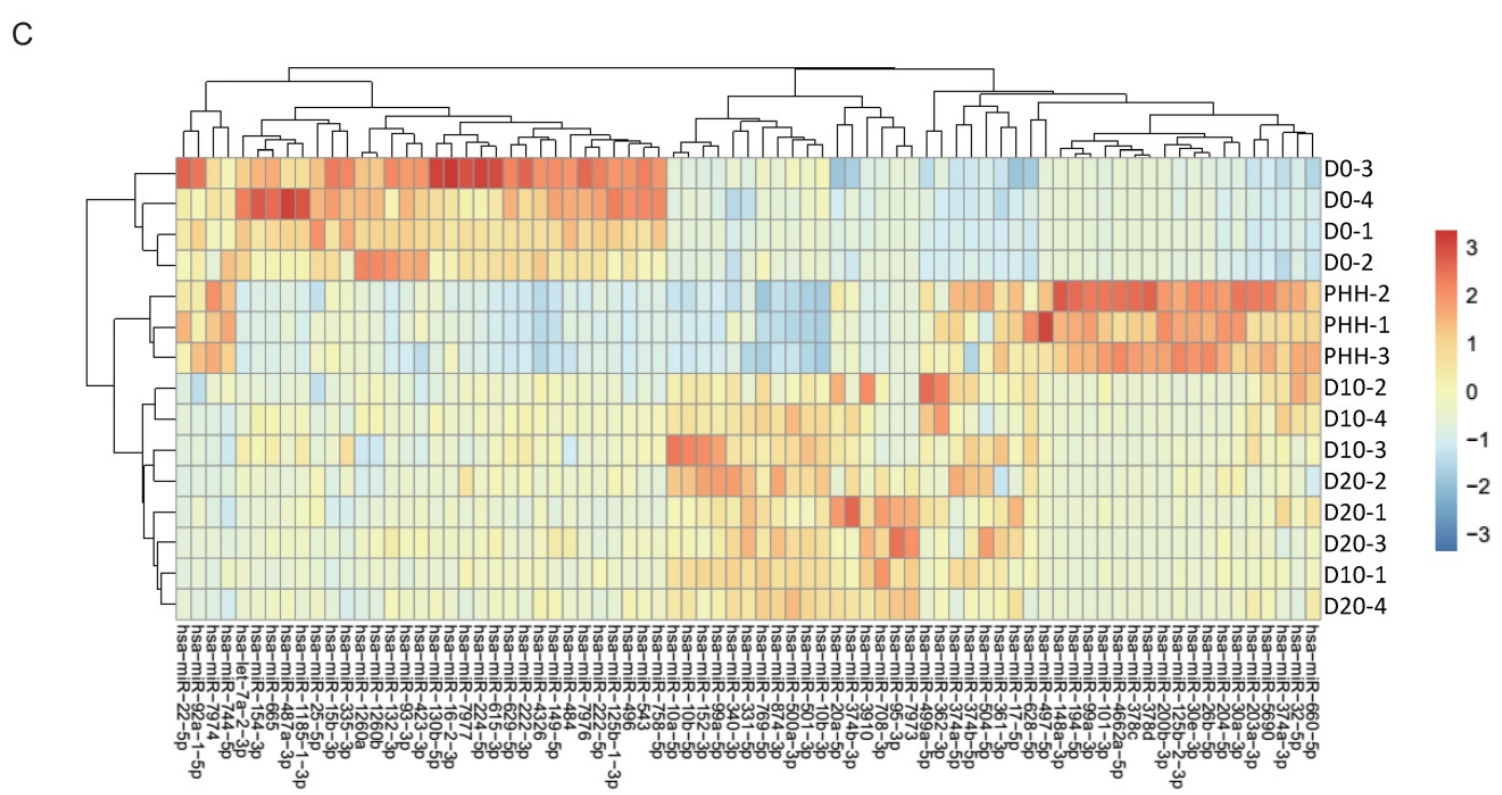

Figure 2. (A) Percentages of the top miRNAs in each sample as determined by sequencing. (B) Significantly differential expression of miRNAs between D10 and D0 is indicated with red lines, and that between D20 and D0 is indicated with blue lines; 77 of the miRNAs shared in both comparisons are indicated with green lines. (C) Clustering results of the samples and miRNAs using 77 differentially expressed miRNAs in undifferentiated hBMSCs (D0), hBMSC-Heps (D10 and D20) and PHHs. 
(D10 and D20) and undifferentiated hBMSCs (D0) was analyzed using DESeq2 v1.8.1. A set of 77 significantly differentially expressed miRNAs were obtained, of which 44 were upregulated and 33 were downregulated during hepatic differentiation (Fig. 2B). Clustering analysis based on the normalized expression of these 77 differentially expressed miRNAs revealed that all hBMSC-Heps were more similar to PHHs than undifferentiated hBMSCs (Fig. 2C). Among the 77 differentially expressed miRNAs, six miRNAs were in the top 10 significantly differentially expressed miRNAs when both the hBMSC-Hep groups (D10 and D20) were compared with the hBMSC group (Fig. 3A). Among the six miRNAs, four miRNAs that showed continuously increasing (hsa-miR-203a-3p, hsa-miR-204-5p, and hsa-miR-101-3p) or decreasing (hsa-miR-93-3p) trends from hBMSCs (D0) to hBMSC-Heps (D10 and D20) to PHHs were selected for subsequent testing. Furthermore, among the 77 differentially expressed
miRNAs, the top 10 most abundant miRNAs in hBMSC-Heps at D20 were selected for additional clustering analysis (Fig. 3B). Three gradually upregulated miRNAs (hsa-miR-148a-3p, hsa-miR101-3p, and hsa-miR-26b-5p) and another three downregulated miRNAs (hsa-miR-423-3p, hsa-miR222-3p, and hsa-miR-224-5p) were selected for subsequent analysis. Overall, nine miRNAs showed gradually changing trends among the top 10 significantly differentially expressed and top 10 most abundant miRNAs were selected for validation.

\section{Validation of the hBMSC-Heps miRNA expression in vitro and in vivo}

To validate the expression levels of the nine selected significantly differentially expressed miRNAs, we performed probe-based qRT-PCR in vitro with cultured hBMSC-Heps and in vivo with tissues from an hBMSC transplantation model in FHF pigs. The changing trends of the nine miRNAs in the four
A

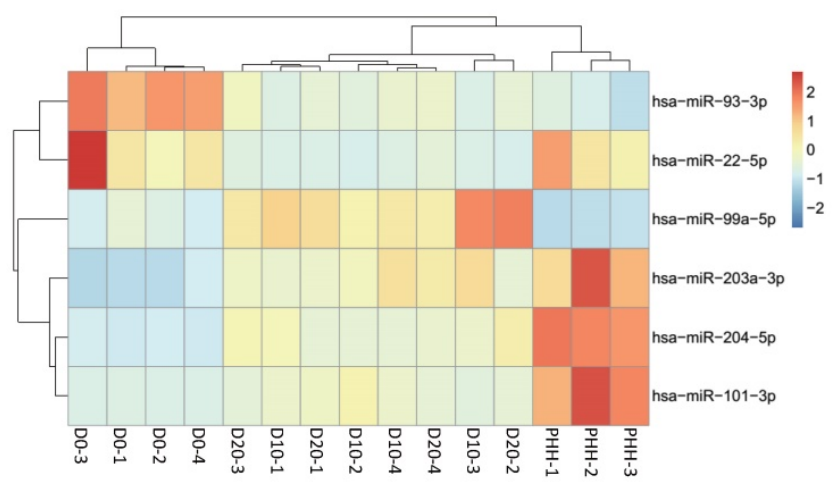

B

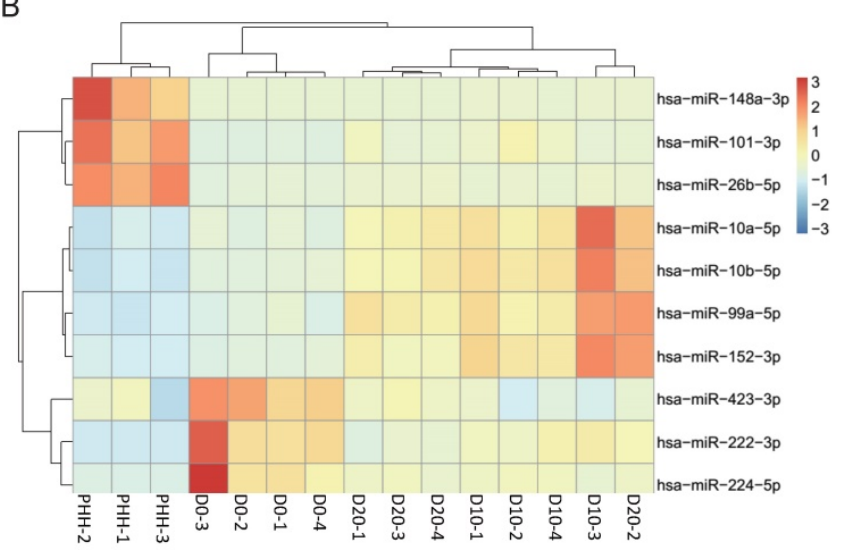

C
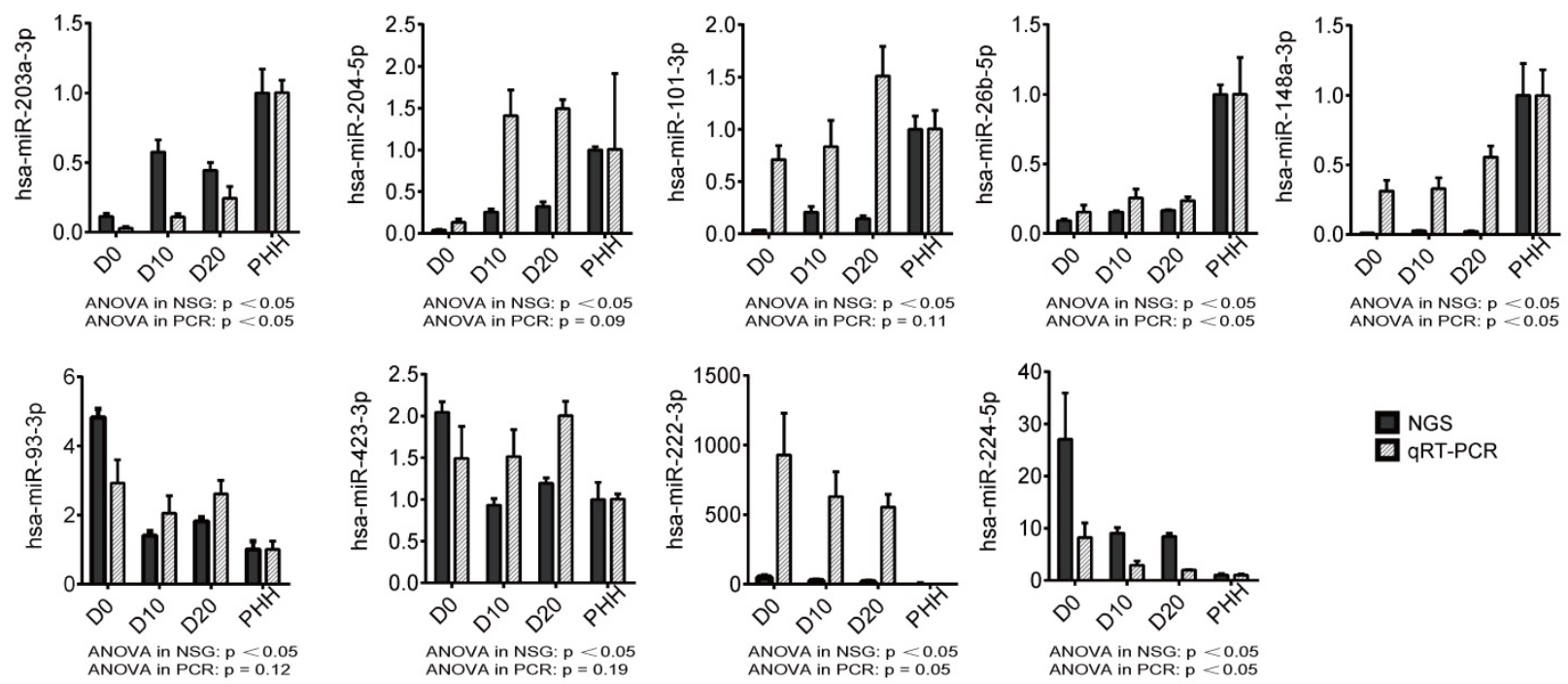

Figure 3. (A) Heatmap of 6 of the top 10 miRNAs significantly differentially expressed between D0 and D10/D20. (B) Heatmap of the top 10 most abundant miRNAs from the set of 77 differentially expressed miRNAs. (C) The changing trends of nine miRNAs in the four cell groups were measured by next-generation sequencing (NGS) and qRT-PCR. $\mathrm{n}=4$ in D0/D10/D20 group, $\mathrm{n}=3$ in $\mathrm{HH}$ group. 
cell groups, as measured by sequencing, were confirmed by qRT-PCR analysis (Fig. 3C). The results showed that three gradually upregulated miRNAs (hsa-miR-203a-3p, hsa-miR-148a-3p, and hsa-miR$26 b-5 p$ ) and another downregulated miRNA (hsamiR-224-5p) were significantly changed $(p<0.05)$.

Our previous study revealed that implanted hBMSCs can quickly differentiate into hepatocyte-like cells in vivo and rescue FHF pigs [9]. To characterize the role of miRNAs during the proliferation and differentiation of hBMSCs into hepatocyte-like cells in vivo, we validated the expression of the nine selected miRNAs in liver tissues using qRT-PCR. All five animals without transplantation died within 4 days, and 4 of $5(80 \%)$ pigs survived beyond five weeks after transplantation. Biochemical analysis of Alanine aminotransferase (ALT), ALB and total bilirubin (TB) showed that the liver function of the transplantation group was much better than that of the control group (Fig. 4A). Analysis of the binding of human-specific antibodies $\left(\mathrm{ALB}^{+}\right.$and hepatocyte-specific antibody $\left.(\mathrm{HSA})^{+}\right)$by immunohistochemistry showed chimerization of human-derived hepatocyte-like cells in the pig liver tissues at weeks 3 and 5 (Fig. 4B) with normal pigs as normal control group (PN). H\&E staining confirmed that pigs subjected to transplantation at week 3 (PT-3W) group and at week 5 (PT-5W) group showed repair of the damaged liver structure, whereas in FHF control group at day 3 after D-galactosamine induction (PC-D3) showed typical FHF histology with extensive hepatic necrosis and hemorrhage (Fig. 4C).

For qRT-PCR validation of the nine selected miRNAs, liver tissues were collected from pigs in the four groups, including two transplantation group (PT-3W, PT-5w) and the normal control PN group with the disease control PC-D3 group. The results of qRT-PCR analysis showed that the changing trends of the nine selected miRNAs in implanted hBMSCs during proliferation and differentiation in vivo were
A
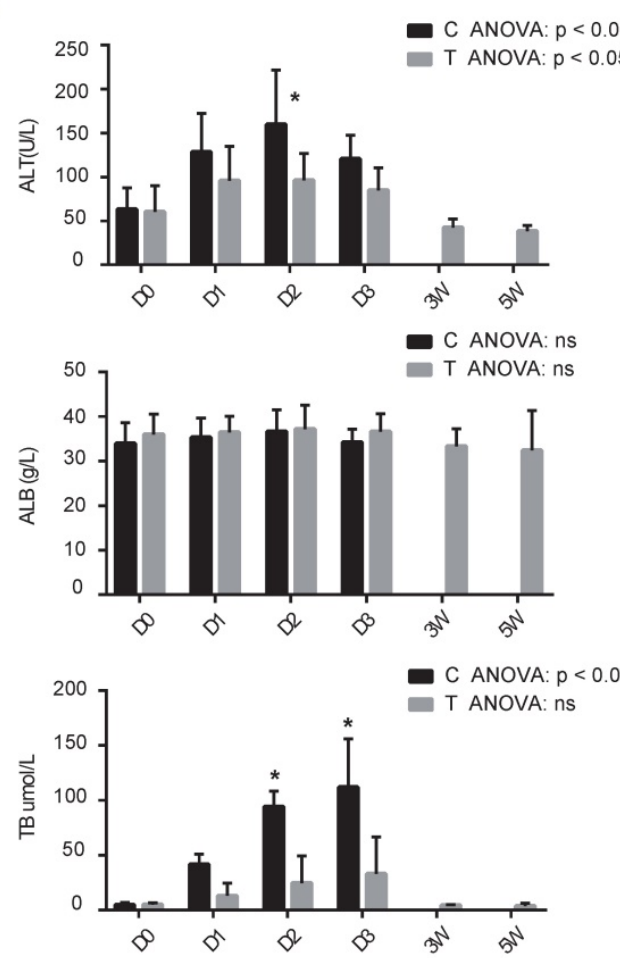

C

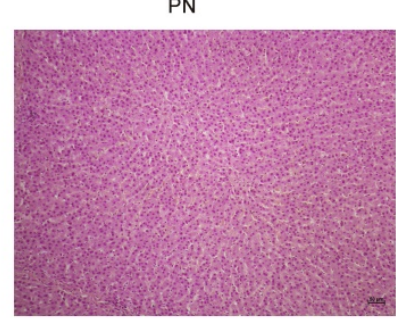

B
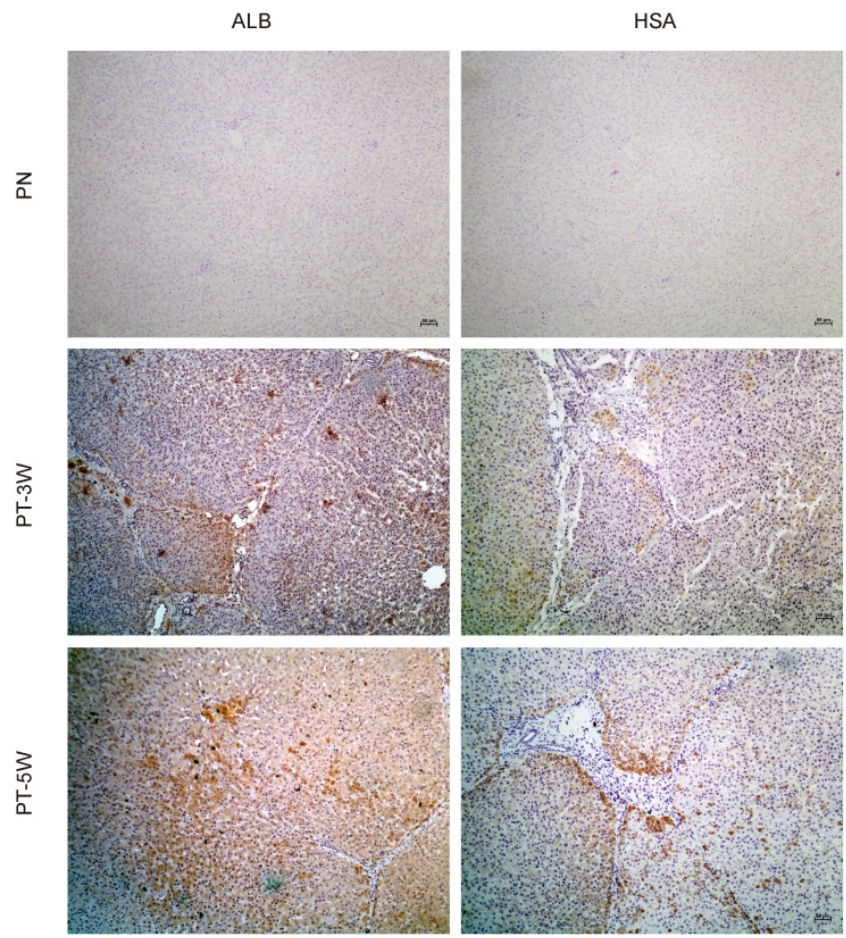

PT-3W
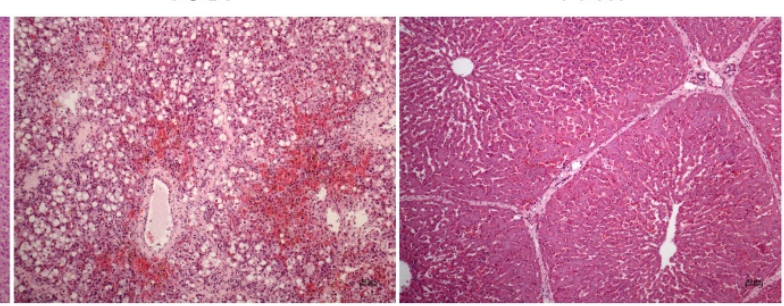

PT-5W

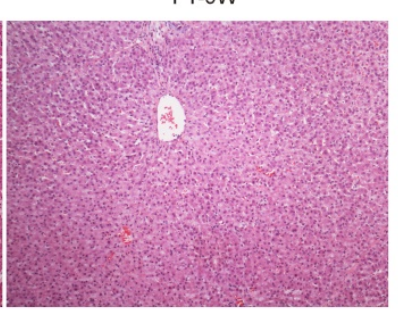

Figure 4. (A) Biochemical analysis of alanine aminotransferase (ALT), albumin (ALB) and total bilirubin (TB) in control group (C) and transplantation group (T), $n=4 / g r o u p . ~(B)$ Immunohistochemistry images of pig livers from the PN, PT-3W and PT-5W groups. Human ALB- and HSA-positive hepatocytes were widely distributed in the hepatic lobules in the PT-3W and PT-5W groups. Scale bar: $50 \mu \mathrm{m}$. (C) H\&E staining of pig liver tissues in the PN, PC-D3, PT-3W and PT-5W groups; scale bar: $50 \mu \mathrm{m}$. 
consistent with those of the miRNAs in cultured hBMSCs undergoing hepatic differentiation (Fig. 5A). The levels of hsa-miR-203a-3p, hsa-miR-204-5p, hsa-miR-101-3p, hsa-miR-26b-5p, hsa-miR-148a-3p hsa-miR-93-3p, hsa-miR-423-3p and hsa-miR-222-3p were much higher in the PT- $5 \mathrm{~W}$ group than in the PC-3D group $(p<0.05)$ except of hsa-miR-224-5p, indicating that the expression of these miRNAs was induced by hBMSC transplantation. Furthermore, the expression of hsa-miR-26b-5p, hsa-miR-148a-3p and hsa-miR-423-3p showed no significant difference when compared with the PN group, indicating that the expression levels were restored to their baseline levels in the PT-5W group after hBMSCs transplantation.

\section{Functional characterization of the selected miRNAs during the differentiation of hBMSCs into hepatocyte-like cells with miRNA activators or miRNA inhibitors}

The above data suggested that the selected
miRNAs are critical regulators during hepatic differentiation. To clarify the roles of these nine miRNAs, we transfected hBMSCs with miRNA activators or miRNA inhibitors for functional studies. Improvement in hepatic differentiation was indicated by the expression of the hepatocyte-specific markers ALB and TAT. Overexpression of hsa-miR-26b-5p and hsa-miR-148a-3p, as well as inhibition of hsa-miR423-3p, showed the capacity to enhance hepatic differentiation, as indicated by increased ALB and TAT expression in the transfected cells (Fig. 5B). The function of a miRNA is ultimately performed by the target mRNAs whose expression it regulates. To gain a better understanding of the role of miRNAs in the proliferation and differentiation of hBMSCs, we searched for the targets of the differentially expressed miRNAs in miRDB. For three functional miRNAs (hsa-miR-26b-5p, hsa-miR-148a-3p and hsa-miR423-3p), 1044 targets were obtained from miRDB. There were 542 liver specific mRNAs among the 1044 targets existed in the data sets of human hepatocytes.
A
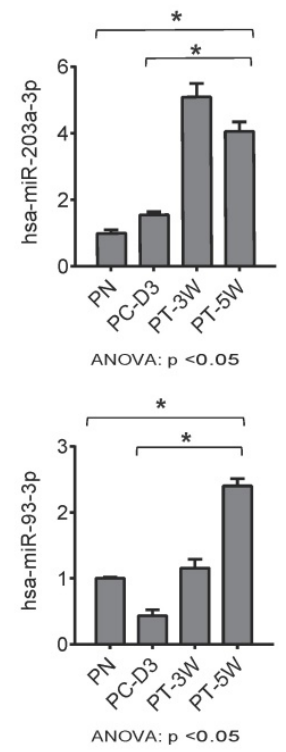

B

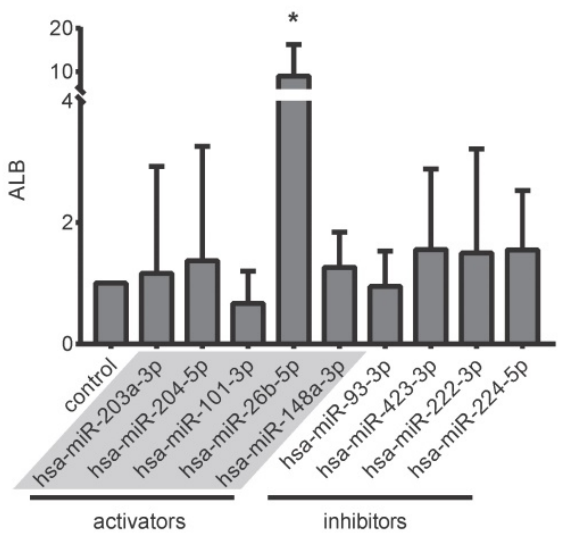

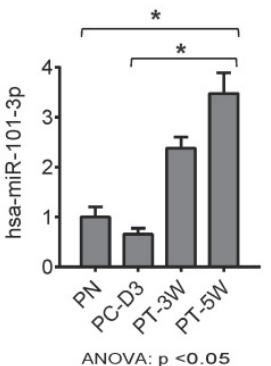
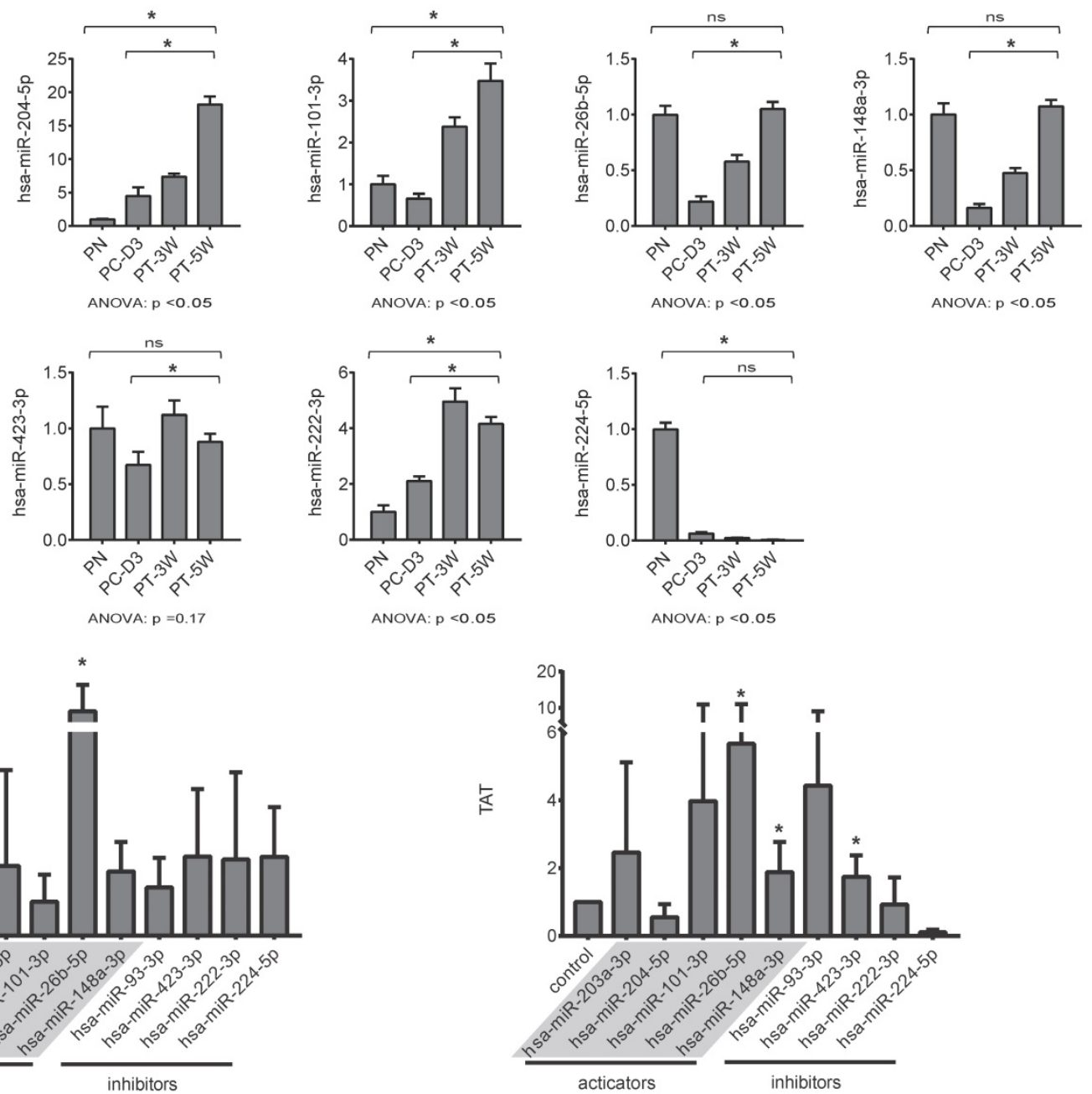

Figure 5. (A) The changing trends of nine miRNAs in the four liver tissue groups were measured by $q R T-P C R, n=4 /$ group. (B) hBMSCs were transfected with miRNA inhibitors or activators and subsequently analyzed for expression of the hepatic markers ALB and TAT by qRT-PCR, $n=5 /$ group. The control column was $h B M S C$-Heps from the standard differentiation protocol. 
To clarify the functions of these 542 mRNAs, clustering analysis of their expression patterns was performed. GO enrichment analysis was used to systematically describe the relationships among the mRNAs and their possible biological mechanisms. Ultimately, $250 \mathrm{GO}$ terms were identified and clustered into $24 \mathrm{GO}$ functional groups (Fig. 6). The main terms with significant enrichment among the genes were related to the characteristics of stem cells, such as cell differentiation, developmental process, and several processes were related to hepatocyte functions, such as protein modification process, metabolic process and catabolic process.
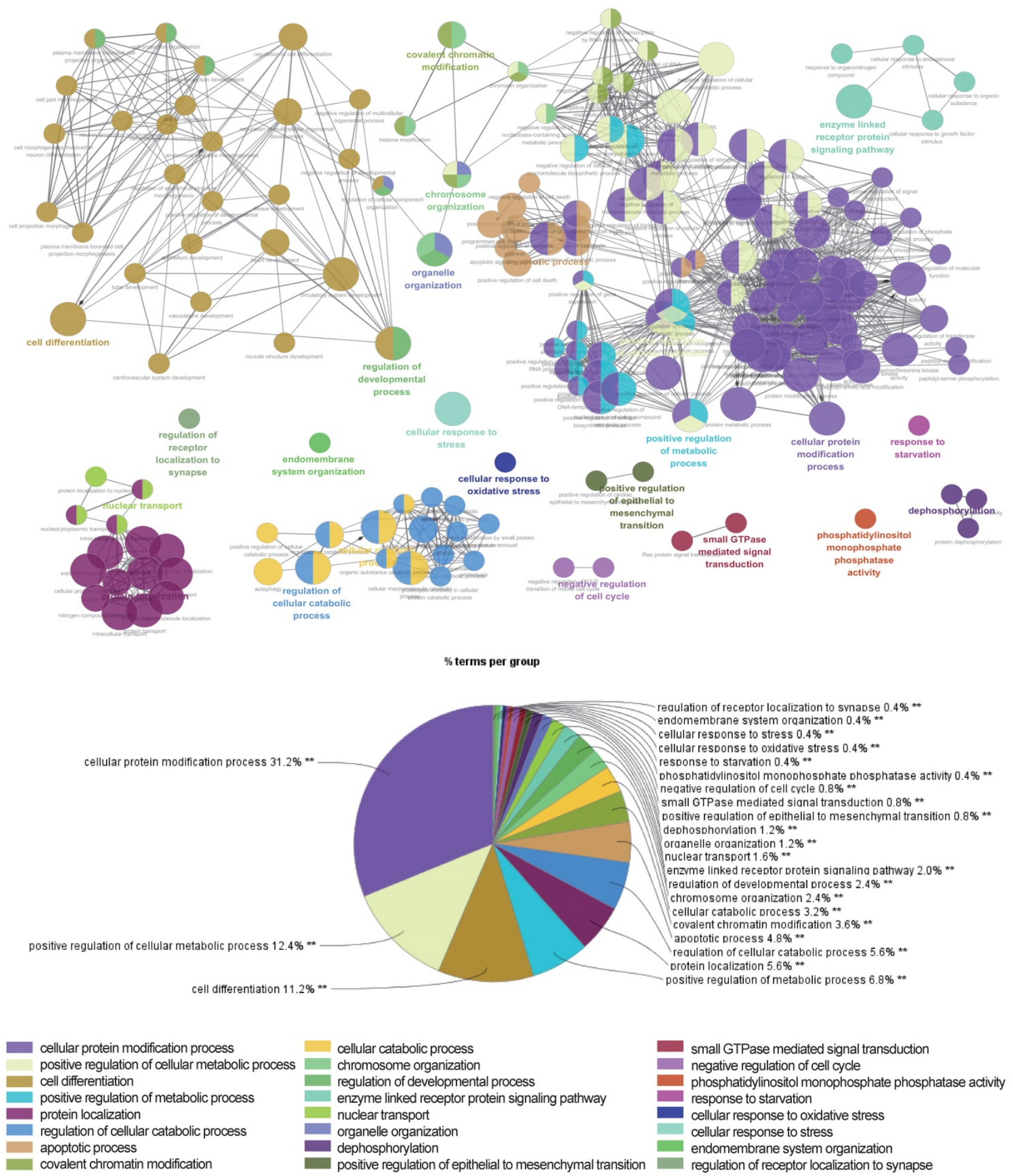

Figure 6. Network of representative GO terms of $\mathbf{5 4 2}$ mRNAs. Each node represents a GO term or a pathway. The colors of the node represent the GO functional groups with the group names showed in the list below the network. The terms are connected based on shared genes. 


\section{Discussion}

Stem cell-based strategies have been the most promising approach for patients suffering from end-stage liver disease and FHF. The in vitro hepatic differentiation process of hBMSCs has been characterized in many studies from our group and others. However, the regulatory mechanism, the efficacy of hBMSC-Heps compared with hepatocytes, and the risk of in vivo application remain unclear.

Mesenchymal stem cells have multipotent differentiation capacity; they can differentiate into multiple types of cells under specific circumstances. In addition to analysis of phenotypic changes, several classic biochemical molecules (e.g., ALB, TAT and TDO2) have been used as biomarkers to evaluate hBMSC-Heps. Previous studies have revealed that each type of stem cells differentiation can be represented by specific miRNA markers [25]. However, the transcriptional and translational expression patterns that hBMSCs present during different developmental stages and the factors that determine the differentiation pattern changes remain unclear. Our results of miRNA profiling at the molecular level revealed the activation of signaling pathways during stem cells hepatic differentiation. The results also indicated the extent to which stem cells differentiate into hBMSC-Heps and enabled comprehensive functional characterization of hBMSC-Heps compared to PHHs. MicroRNA-148a has been reported as a key molecule during liver development, as its expression is higher in the fetal liver than in the adult liver. In this study, the expression of hsa-miR-148a-3p rapidly increased during the hepatic differentiation of hBMSCs, but the level was still lower than its expression in PHHs. Furthermore, the most abundantly expressed miRNA during stem cells hepatic differentiation, hsa-miR-21-5p, played an important role in hepatic proliferation, but its expression remained high in hBMSC-Heps. In contrast, the liver-specific miRNA hsa-miR-122-5p, which accounted for $\sim 20 \%$ of all expressed miRNAs in PHHs, did not accumulate to appreciable levels in hBMSC-Heps. These results showed that the differentiation of stem cells in vitro was still not complete.

In vivo, stem cells or progenitor cells contribute to supplying hepatocytes for the maintenance of liver homeostasis and for recovery from injury. In this study, the expression levels of nine selected miRNAs were tracked by qRT-PCR in liver tissues from FHF pigs receiving hBMSC transplantation. The levels of the functional miRNAs indicated a significant difference in hepatic regeneration function in the PT- $3 W$ group compared to the PC-3D group; the levels returned to baseline in the PT-5W group and were similar to the levels in the $\mathrm{PN}$ group. Our confirmation of the miRNA sequences and the qRT-PCR results demonstrated that the selected miRNAs could be important regulators during stem cells transplantation. The above results imply that the current protocols for hepatic differentiation of hBMSCs successfully provide hBMSC-derived functional hepatocyte-like cells but still need substantial improvements. Understanding the hepatic differentiation of hBMSCs in vivo yields insight into molecules that can be used to instruct stem cells to differentiate and grow.

The function of miRNAs in controlling hepatocyte proliferation during liver regeneration and in improving the differentiation of hepatic precursor cells has been reported [31,32,33], but the mechanism needs to be elucidated [34]. In this study, miRNA activators and miRNA inhibitors were used for gain-of-function or loss-of-function studies. The results showed that hsa-miR-26b-5p, hsa-miR-148a-3p and hsa-miR-423-3p played key roles in hBMSC hepatic differentiation and promoted hepatic gene expression during differentiation. MicroRNA-148a has been reported to be a regulator of the low-density lipoprotein (LDL) receptor [35] and can promote hepatic lipid metabolism in mice [36]; it is also one major member of a set of six miRNAs mediating the induction of human umbilical cord lining-derived mesenchymal stem cell (MSC) differentiation into hepatocyte-like cells. Other studies have indicated that MicroRNA-26b participates in regulating the chemotactic response of MSCs toward HGF [37] and regulates proliferation, angiogenesis and apoptosis in hepatocellular carcinoma [38]. Furthermore, recent studies have determined the role and mechanism of miR-423-5p in hepatic glucose and lipid metabolism [39]. These results indicate that hsa-miR-26b-5p, hsa-miR-148a-3p and hsa-miR-423-3p could play an essential role in hepatic differentiation of hBMSCs. While a single mRNA can be regulated by several miRNAs, it is difficult to elevate hepatic function completely by a single miRNA, so the combined manipulation of miRNAs provides a new perspective to enhance stem cells differentiation. Considering the functions of miRNAs in inducing the proliferation of mature hepatocytes and improving the differentiation of hBMSCs, miRNA-based technologies provide attractive tools for improving therapeutic approaches involving hBMSCs.

\section{Conclusions}

Our study identified the miRNA profile of hBMSCs in several hepatic differentiation states and validated the miRNA basis of hBMSC-Heps in vitro 
and in vivo through hBMSC transplantation into FHF pigs. Due to the powerful ability of miRNAs to fine-tune biological processes relevant to hepatic differentiation, developmental growth and cell functions, our discovery of specific miRNAs may enable significant improvements in the evaluation and acceleration of hBMSC differentiation into hepatocytes and may lead to beneficial clinical applications in the future.

\section{Abbreviations}

ALB: albumin; ALT: alanine aminotransferase; FHF: fulminant hepatic failure; GO: Gene ontology; hBMSCs: human bone marrow mesenchymal stem cells; hBMSC-Heps: hBMSC-differentiated hepatocyte-like cells; hHGF: human hepatocyte growth factor; HSA: hepatocyte-specific antibody; H\&E: Hematoxylin and eosin; ITS: insulin, transferrin, and selenium; miRNAs: microRNAs; OSM: oncostatin M; PAS: periodic acid-Schiff; PHHs: primary human hepatocytes; qRT-PCR: quantitative real-time polymerase chain reaction; TAT: tyrosine aminotransferase; TB: total bilirubin; TDO2: tryptophan 2,3-dioxygenase.

\section{Supplementary Material}

Supplementary tables.

https://www.medsci.org/v19p0152s1.pdf

\section{Acknowledgements}

\section{Funding}

This work was supported by the National Natural Science Foundation of China (31700851/ 81571818/81771996/81901901), National S\&T Major Project (2017ZX10203201/2017ZX10304402), Zhejiang Public Welfare Project (LGF21H200006/2017C37072), Zhejiang Provincial Key R\&D Program (2017C01046/2017C01026) and Independent Project Fund of the State Key Laboratory for Diagnosis and Treatment of Infectious Disease.

\section{Declarations}

The study was approved by the Ethics Committee of the First Affiliated Hospital, Zhejiang University School of Medicine (No. 2011013).

\section{Availability of data and material}

Sequencing reads from our miRNA-seq experiments are available in the Sequence Read Archive database (accession number: PRJNA613229).

\section{Author Contributions}

JJ and JJX performed the experiments and wrote the main manuscript. JJ and WCD analyzed the data. SWS and DYS did part of the cellular experiment. BBG,
XPZ and CFZ helped with the stem cells collection. JL conceived and designed the experiments. All authors read and approved the final manuscript.

\section{Competing Interests}

The authors have declared that no competing interest exists.

\section{References}

1. Bernal W, Jalan R, Quaglia A, Simpson K, Wendon J, Burroughs A. Acute-on-chronic liver failure. Lancet 2015, 386(10003): 1576-1587.

2. Elias E. Liver failure and liver disease. Hepatology 2006, 43(2 Suppl 1): S239-242.

3. Starzl TE. The long reach of liver transplantation. Nature medicine 2012, 18(10): 1489-1492.

4. Christ B, Bruckner S, Winkler S. The Therapeutic Promise of Mesenchymal Stem Cells for Liver Restoration. Trends in molecular medicine 2015, 21(11): 673-686.

5. Duncan AW, Dorrell C, Grompe M. Stem cells and liver regeneration. Gastroenterology 2009, 137(2): 466-481.

6. Miyajima A, Tanaka M, Itoh T. Stem/progenitor cells in liver development, homeostasis, regeneration, and reprogramming. Cell stem cell 2014, 14(5): 561-574

7. Kuroda Y, Kitada M, Wakao S, Dezawa M. Bone marrow mesenchymal cells: how do they contribute to tissue repair and are they really stem cells? Archivum immunologiae et therapiae experimentalis 2011, 59(5): 369-378.

8. Li J, Tao R, Wu W, Cao H, Xin J, Guo J, et al. Transcriptional profiling and hepatogenic potential of acute hepatic failure-derived bone marrow mesenchymal stem cells. Differentiation; research in biological diversity 2010, 80(2-3): 166-174

9. Li J, Zhang L, Xin J, Jiang L, Li J, Zhang T, et al. Immediate intraportal transplantation of human bone marrow mesenchymal stem cells prevents death from fulminant hepatic failure in pigs. Hepatology 2012, 56(3): 1044-1052.

10. Lin BL, Chen JF, Qiu WH, Wang KW, Xie DY, Chen XY, et al. Allogeneic bone marrow-derived mesenchymal stromal cells for hepatitis B virus-related acute-on-chronic liver failure: A randomized controlled trial. Hepatology 2017, 66(1): 209-219.

11. Bhatia SN, Underhill GH, Zaret KS, Fox IJ. Cell and tissue engineering for liver disease. Science translational medicine 2014, 6(245): 245sr242.

12. Carthew RW, Sontheimer EJ. Origins and Mechanisms of miRNAs and siRNAs. Cell 2009, 136(4): 642-655.

13. Rana TM. Illuminating the silence: understanding the structure and function of small RNAs. Nature reviews Molecular cell biology 2007, 8(1): 23-36.

14. Guo Z, Maki M, Ding R, Yang Y, Zhang B, Xiong L. Genome-wide survey of tissue-specific microRNA and transcription factor regulatory networks in 12 tissues. Scientific reports 2014, 4: 5150

15. Lagos-Quintana M, Rauhut R, Yalcin A, Meyer J, Lendeckel W, Tuschl T. Identification of tissue-specific microRNAs from mouse. Current biology : CB 2002, 12(9): 735-739.

16. Shenoy A, Blelloch RH. Regulation of microRNA function in somatic stem cell proliferation and differentiation. Nature reviews Molecular cell biology 2014, 15(9): 565-576.

17. Tzur G, Israel A, Levy A, Benjamin H, Meiri E, Shufaro Y, et al. Comprehensive gene and microRNA expression profiling reveals a role for microRNAs in human liver development. PloS one 2009, 4(10): e7511.

18. Liu D, Fan J, Zeng W, Zhou Y, Ingvarsson S, Chen H. Quantitative analysis of miRNA expression in several developmental stages of human livers. Hepatology research: the official journal of the Japan Society of Hepatology 2010, 40(8): 813-822.

19. Song G, Sharma AD, Roll GR, Ng R, Lee AY, Blelloch RH, et al. MicroRNAs control hepatocyte proliferation during liver regeneration. Hepatology 2010, 51(5): 1735-1743.

20. Gangaraju VK, Lin H. MicroRNAs: key regulators of stem cells. Nature reviews Molecular cell biology 2009, 10(2): 116-125.

21. Rogler CE, Levoci L, Ader T, Massimi A, Tchaikovskaya T, Norel R, et al. MicroRNA-23b cluster microRNAs regulate transforming growth factor-beta/bone morphogenetic protein signaling and liver stem cell differentiation by targeting Smads. Hepatology 2009, 50(2): 575-584.

22. Doddapaneni R, Chawla YK, Das A, Kalra JK, Ghosh S, Chakraborti A. Overexpression of microRNA-122 enhances in vitro hepatic differentiation of fetal liver-derived stem/ progenitor cells. Journal of cellular biochemistry 2013, 114(7): 1575-1583.

23. Deng XG, Qiu RL, Wu YH, Li ZX, Xie P, Zhang J, et al. Overexpression of miR-122 promotes the hepatic differentiation and maturation of mouse ESCs through a miR-122/FoxA1/HNF4a-positive feedback loop. Liver international : official journal of the International Association for the Study of the Liver 2014, 34(2): 281-295.

24. Kim N, Kim H, Jung I, Kim Y, Kim D, Han YM. Expression profiles of miRNAs in human embryonic stem cells during hepatocyte differentiation. Hepatology 
research: the official journal of the Japan Society of Hepatology 2011, 41(2): 170-183.

25. Cui L, Zhou X, Li J, Wang L, Wang J, Li Q, et al. Dynamic microRNA profiles of hepatic differentiated human umbilical cord lining-derived mesenchymal stem cells. PloS one 2012, 7(9): e44737.

26. Xin J, Ding $\mathrm{W}$, Hao S, Jiang L, Zhou $\mathrm{Q}, \mathrm{Wu} \mathrm{T}$, et al. Human bone marrow mesenchymal stem cell-derived hepatocytes express tissue inhibitor of metalloproteinases 4 and follistatin. Liver international: official journal of the International Association for the Study of the Liver 2015, 35(10): 2301-2310.

27. Li J, Xin JJ, Zhang LY, Wu J, Jiang LY, Zhou Q, et al. Human Hepatic Progenitor Cells Express Hematopoietic Cell Markers CD45 and CD109. Int J Med Sci 2014, 11(1): 65-79.

28. Friedlander MR, Mackowiak SD, Li N, Chen W, Rajewsky N. miRDeep2 accurately identifies known and hundreds of novel microRNA genes in seven animal clades. Nucleic acids research 2012, 40(1): 37-52.

29. Ding W, Xin J, Jiang L, Zhou Q, Wu T, Shi D, et al. Characterisation of peripheral blood mononuclear cell microRNA in hepatitis B-related acute-on-chronic liver failure. Scientific reports 2015, 5: 13098.

30. Bindea G, Mlecnik B, Hackl H, Charoentong P, Tosolini M, Kirilovsky A, et al. ClueGO: a Cytoscape plug-in to decipher functionally grouped gene ontology and pathway annotation networks. Bioinformatics 2009, 25(8): 1091-1093.

31. Mobus S, Yang D, Yuan Q, Ludtke TH, Balakrishnan A, Sgodda M, et al. MicroRNA-199a-5p inhibition enhances the liver repopulation ability of human embryonic stem cell-derived hepatic cells. Journal of hepatology 2015, 62(1): 101-110.

32. Yuan Q, Loya K, Rani B, Mobus S, Balakrishnan A, Lamle J, et al. MicroRNA-221 overexpression accelerates hepatocyte proliferation during liver regeneration. Hepatology 2013, 57(1): 299-310.

33. Cui L, Shi $Y$, Zhou X, Wang X, Wang J, Lan Y, et al. A set of microRNAs mediate direct conversion of human umbilical cord lining-derived mesenchymal stem cells into hepatocytes. Cell death \& disease 2013, 4: e918.

34. Raschzok N, Sallmon H, Pratschke J, Sauer IM. MicroRNAs in liver tissue engineering - New promises for failing organs. Advanced drug delivery reviews 2015, 88: 67-77.

35. Goedeke L, Rotllan N, Canfran-Duque A, Aranda JF, Ramirez CM, Araldi E, et al. MicroRNA-148a regulates LDL receptor and ABCA1 expression to control circulating lipoprotein levels. Nature medicine 2015, 21(11): 1280-1289.

36. Cheng L, Zhu Y, Han H, Zhang Q, Cui K, Shen H, et al. MicroRNA-148a deficiency promotes hepatic lipid metabolism and hepatocarcinogenesis in mice. Cell death \& disease 2017, 8(7): e2916.

37. Zhu A, Kang N, He L, Li X, Xu X, Zhang H. MiR-221 and miR-26b Regulate Chemotactic Migration of MSCs Toward HGF Through Activation of Akt and FAK. Journal of cellular biochemistry 2016, 117(6): 1370-1383.

38. Wang $\mathrm{Y}$, Sun B, Sun $\mathrm{H}$, Zhao X, Wang X, Zhao N, et al. Regulation of proliferation, angiogenesis and apoptosis in hepatocellular carcinoma by miR-26b-5p. Tumour biology: the journal of the International Society for Oncodevelopmental Biology and Medicine 2016, 37(8): 10965-10979.

39. Yang $W$, Wang $J$, Chen $Z$, Chen J, Meng $Y$, Chen $L$ et al. NFE2 Induces miR-423-5p to Promote Gluconeogenesis and Hyperglycemia by Repressing the Hepatic FAM3A-ATP-Akt Pathway. Diabetes 2017, 66(7): 1819-1832. 